\title{
Safety of Pilocarpine Therapy in Patients With Sjögren's Syndrome: A Single-Arm Interventional, Post-Market Surveillance Study
}

\author{
Deng-Ho Yang ${ }^{1-4}$, Feng-Cheng Liu ${ }^{4}$, Chun-Chi Lu ${ }^{4}$, San-Yuan $\mathrm{Kuo}^{4}$, Shi-Jye $\mathrm{Chu}^{4}$, and Hsiang-Cheng Chen*4 \\ ${ }^{1}$ Division of Rheumatology/Immunology/Allergy, Department of Internal Medicine, Taichung Armed-Forces General Hospital, Taichung, Taiwan \\ ${ }^{2}$ Department of Laboratory, Taichung Armed Forces General Hospital, Taichung, Taiwan
}

${ }^{3}$ Department of Medical Laboratory Science and Biotechnology, Central Taiwan University of Science and Technology, Taichung, Taiwan

${ }^{4}$ Division of Rheumatology/Immunology/Allergy, Department of Internal Medicine, Tri-Service General Hospital, National Defense Medical Center, Taipei, Taiwan

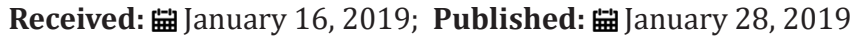

*Corresponding author: Hsiang-Cheng Chen, Division of Rheumatology/Immunology/Allergy, Department of Internal Medicine, TriService General Hospital, No. 325, Sec. 2, Cheng-Kung Rd, Neihu 114, Taipei, Taiwan

\begin{abstract}
Sjögren's syndrome is a systemic immune disease with impaired secretion of salivary glands; thus, it may cause inconvenience in patients' daily lives. Pilocarpine is a non-selective muscarinic receptor agonist used to treat dry mouth. This single-arm intervention, post-market surveillance study evaluated the safety of pilocarpine (Salicret, Meider Pharmaceutical Co., Ltd.) for treating Sjögren's syndrome. We recruited 135 patients with Sjögren's syndrome who received pilocarpine orally four times daily at a dose of $5 \mathrm{mg}$ for 24 weeks. Forty-one (31.3\%) of 131 patients experienced at least one adverse event (AE), and of those, 37 patients' (28.2\%) AEs were associated with the study treatment. The most common drug-related AEs were sweating in 14 patients $(10.7 \%)$ and palpitation in 9 (6.9\%). No serious AEs occurred during the study period. Therefore, pilocarpine therapy is considered effective, safe, and well tolerated in patients with Sjögren's syndrome.
\end{abstract}

Keywords: Pilocarpine; Salicret; Sjögren’s Syndrome; Adverse Event

\section{Introduction}

The functions of saliva include lubricating the mouth, aiding in the digestion process, and possessing antimicrobial functions [1]. Xerostomia is dryness of the mouth that may be associated with a change in the composition of saliva or reduced salivary flow [2]. Dehydration, malfunction of the salivary glands, or radiotherapy of the area of the salivary glands can cause xerostomia. The general symptoms of xerostomia include a persistent oral burning sensation, eating difficulties, diminution in taste acuity, discomfort speaking, recurrent mucosal infections, greater periodontal disease, and denture intolerance [3]. Xerostomia affects the mechanical process of providing moisture and lubricating the mouth with saliva, and the chemical, antimicrobial, remineralizing, and buffering functions of saliva [4]. A reduction in salivation is paralleled by a shift to highly cariogenic microflora at the expense of non-cariogenic organisms [5]. Therefore, saliva plays important roles in protecting the oral cavity. Sjögren's syndrome is one of the causes of xerostomia with destructive salivary and lacrimal glands due to abnormal infiltration of lymphocytes [6]. The attack on these exocrine glands may lead to the development of xerostomia and keratoconjunctivitis sicca.
The autoimmune response to Sjögren's syndrome is associated with lymphocytic infiltration that eventually causes severe damage or destruction of the glands. Clinically, there are two classes of Sjögren's syndrome: primary and secondary types. Primary Sjögren's syndrome occurs independently, whereas secondary Sjögren's syndrome occurs concomitantly with other connective tissue diseases [7]. Primary Sjögren's syndrome is a common systemic autoimmune disease with a prevalence of $0.05-4.8 \%$ [8]. Its incidence in women is approximately 9 times higher than that in men [9]. Secondary Sjögren's syndrome may be associated with rheumatoid arthritis, systemic lupus erythematosus, systemic sclerosis, multiple sclerosis, autoimmune thyroiditis, and autoimmune hepatitis [10]. Patients with primary or secondary Sjögren's syndrome have the same level of discomfort, complications, and severity of disease. Oral lesions including angular cheilitis, atrophic glossitis, recurrent ulcerations, and fissurations of the tongue are usually found [11]. The pathogenesis of Sjögren's syndrome is unknown, but it is believed to be caused by 
genetic predisposition, environmental and hormonal factors, and a disordered immune system $[6,12]$.

The clinical symptoms including dryness of the mouth and eyes, fatigue, and joint pain are usually found in $80 \%$ of patients with Sjögren's syndrome [13]. To date, neither a cure for Sjögren's syndrome nor a specific treatment is known to permanently restore gland secretion. Instead, treatment is generally symptomatic and supportive. Adequate immunotherapy may improve the systemic symptoms of Sjögren's syndrome, including arthritis, pain, interstitial pulmonary fibrosis, and neuropathy [10], but immunotherapy has no adequate response for the symptoms of dry mouth or dry eye [14]. However, prescription drugs including cevimeline and pilocarpine are available to help stimulate salivary flow $[10,13,15]$. Pilocarpine is a cholinergic parasympathomimetic alkaloid originally extracted from Jaborandi (Pilocarpus microphyllus) [16]. It is a non-selective muscarinic receptor agonist. Its pharmacological actions can stimulate exocrine glands and result in diaphoresis, salivation, lacrimation, and gastric and pancreatic secretion. Pilocarpine can provide clinically significant symptomatic relief to patients with xerostomia $[17,18]$. Pilocarpine has been approved for the treatment of xerostomia resulting from radiation damage to salivary glands and from Sjögren's syndrome [10,17]. We aimed to evaluate the safety of oral pilocarpine (Salicret, Meider Pharmaceutical Co., Ltd.) for treating patients with Sjögren's syndrome.

\section{Methods}

\section{Study Design and Participants}

This single-arm interventional, post-market surveillance study aimed to recruit 135 patients from February 12, 2015 (first patient, first visit) to March 20, 2017 (last patient, last visit), and it was conducted in Tri-Service General Hospital and Taichung Armed-Forces General Hospital. This study was approved by the Institutional Review Board of Tri-Service General Hospital (TSGHIRB number 2-103-05-140), and all patients in the study provided written informed consent

Table 1: Flow chart of the study.

\begin{tabular}{|c|c|c|c|}
\hline Procedures & Screening and baseline visits & Returning (evaluation) visit & \\
\hline Visit & 1 & 2 & 12 \\
\hline Week & 0 & \pm 7 & \pm 7 \\
\hline Visit window (days) & Not applicable & & \\
\hline Obtain informed consent & $\mathrm{X}$ & & \\
\hline $\begin{array}{c}\text { Assess inclusion and exclusion } \\
\text { criteria }\end{array}$ & $\mathrm{X}$ & & \\
\hline $\begin{array}{c}\text { Record demographic data and } \\
\text { medical history }\end{array}$ & $\mathrm{X}$ & & \\
\hline Record history of medication & $\mathrm{X}$ & & \\
\hline $\begin{array}{c}\text { Perform urine pregnancy test } \\
\text { applicable for women of child- } \\
\text { bearing age) }\end{array}$ & $\mathrm{X}$ & $\mathrm{X}$ & \\
\hline Perform physical examinations & $\mathrm{X}$ & $\mathrm{X}$ & $\mathrm{X}$ \\
\hline Assess vital signs & & \\
\hline Conduct 12-lead ECG & & & \\
\hline
\end{tabular}

Cite this article: Deng-Ho Yang, Feng-Cheng Liu, Chun-Chi Lu, San-Yuan Kuo, Shi-Jye Chu, Hsiang-Cheng Chen. Study of Phototoxicity of LED 


\begin{tabular}{|c|c|c|c|}
\hline Measure body weight & $X$ & $X$ & $X$ \\
\hline Dispense trial medication & $X$ & $X$ & $X$ \\
\hline Record concomitant medications & & $X$ & $X$ \\
\hline Record AEs & & $X$ & $X$ \\
\hline Dismiss patient from study & & & \\
\hline
\end{tabular}

Note: ECG: electrocardiography, AE: adverse event.

\section{Results}

\section{Patients' Characteristics}

One hundred thirty-five patients were enrolled in this study. Based on the most updated version of the protocol, we planned to enroll 160 patients with an expected safety-evaluable population of $80 \%$ to have at least 128 patients for safety analysis. Four patients who did not return for a visit were excluded from the safety analysis; ultimately, 131 patients were included in our safety population. Thirty-nine of 135 patients (28.9\%) withdrew from the study, including 19 patients (14.1\%) because of an unacceptable AE and $20(14.8 \%)$ who withdrew voluntarily. The study population was comprised of 8 men (6.1\%) and 123 women (93.9\%). Patients' average age was 59.0 years (range 21.3-87.2 years). All patients had ocular and oral symptoms at study entry and fulfilled at least four of the six classification criteria of Sjögren's syndrome. Most patients (93.1\%) were diagnosed as having primary Sjögren's syndrome. All patients' characteristics are shown in Table 2.

Table 2: Patients' characteristics.

\begin{tabular}{|c|c|c|}
\hline & Classification criteria of Sjögren's syndrome & (N=131) \\
\hline Symptoms of Sjögren's syndrome & Ocular symptoms & $131(100 \%)$ \\
\hline & Oral symptoms & $105(80.2 \%)$ \\
\hline & Positive Schirmer's test result & $58(44.3 \%)$ \\
\hline & Focus score $\geq 1$ & $84(64.1 \%)$ \\
\hline Diagnosis & Salivary gland involvement & $71(54.2 \%)$ \\
\hline & Positive SSA or SSB & $122(93.1 \%)$ \\
\hline
\end{tabular}

Note: SSA: Sjögren's syndrome A, SSB: Sjögren's syndrome B, SS: Sjögren's syndrome.

\section{Common AEs}

The analysis of safety assessments was performed based on the patients who received at least one dose of study treatment with at least one safety evaluation after receiving study treatment. Overall, 50 AEs were observed in 41 patients (31.3\%), and 45 drug-related AEs were observed in 37 patients (28.2\%) during the study period. The numbers of one or more AEs by severity are presented in Table 3 . The most common AEs observed were sweating (including general sweating, night sweating, cold sweating, and severe sweating) (10.7\%) and palpitation (7.6\%). Other drug-related AEs commonly reported in our patients included diarrhea (3.1\%), dizziness (3.1\%), nausea/sensation of nausea (2.3\%), epigastric irritation/ epigastric discomfort/stomach pain (2.3\%), and headache $(1.5 \%)$. Most drug-related AEs were mild to moderate in severity, except severe sweating occurred in one patient. No serious adverse events (SAEs) occurred during the study period.

Table 3: Number of subjects with one or more adverse events by severity.

\begin{tabular}{|c|c|c|c|c|}
\hline Adverse Event & \multicolumn{2}{|c|}{ Patients treated with pilocarpine (Salicret, Meider Pharmaceutical Co., Ltd.) (N=131) } \\
\hline & Mild & Moderate & Severe & Total \\
\hline Allergic Urticaria & $1(0.8 \%)$ & $0(0 \%)$ & $0(0 \%)$ & $1(0.8 \%)$ \\
\hline Cold Sweating & $0(0 \%)$ & $1(0.8 \%)$ & $0(0 \%)$ & $1(0.8 \%)$ \\
\hline Diarrhea & $4(3.1 \%)$ & $0(0 \%)$ & $0(0 \%)$ & $4(3.1 \%)$ \\
\hline Discomfort of the Legs & $1(0.8 \%)$ & $0(0 \%)$ & $0(0 \%)$ & $1(0.8 \%)$ \\
\hline Dizziness & $3(2.3 \%)$ & $1(0.8 \%)$ & $0(0 \%)$ & $4(3.1 \%)$ \\
\hline Epigastric Discomfort & $1(0.8 \%)$ & $0(0 \%)$ & $0(0 \%)$ & $1(0.8 \%)$ \\
\hline Epigastric Irritation & $0(0 \%)$ & $1(0.8 \%)$ & $0(0 \%)$ & $1(0.8 \%)$ \\
\hline Headache & $2(1.5 \%)$ & $0(0 \%)$ & $0(0 \%)$ & $2(1.5 \%)$ \\
\hline Hypertension & $1(0.8 \%)$ & $0(0 \%)$ & $0(0 \%)$ & $1(0.8 \%)$ \\
\hline Itchy Skin Rash & $1(0.8 \%)$ & $0(0 \%)$ & $1(0.8 \%)$ \\
\hline
\end{tabular}




\begin{tabular}{|c|c|c|c|c|}
\hline Low back pain & $1(0.8 \%)$ & $0(0 \%)$ & $0(0 \%)$ & $1(0.8 \%)$ \\
\hline Malaise & $1(0.8 \%)$ & $0(0 \%)$ & $0(0 \%)$ & $1(0.8 \%)$ \\
\hline Nausea & $2(1.5 \%)$ & $0(0 \%)$ & $0(0 \%)$ & $2(1.5 \%)$ \\
\hline Sensation of Nausea & $1(0.8 \%)$ & $0(0 \%)$ & $0(0 \%)$ & $1(0.8 \%)$ \\
\hline Night Sweating & $1(0.8 \%)$ & $0(0 \%)$ & $0(0 \%)$ & $1(0.8 \%)$ \\
\hline Palpitation & $8(6.1 \%)$ & $0(0 \%)$ & $0(0 \%)$ & $1(0.8 \%)$ \\
\hline Severe Sweating & $1(0.8 \%)$ & $0(0 \%)$ & $1(0 \%)$ & $1(0.8 \%)$ \\
\hline Stomach Pain & $1(0.8 \%)$ & $1(0.8 \%)$ & $1(0.8 \%)$ & $11(8.4 \%)$ \\
\hline General Sweating & $9(6.9 \%)$ & $0(0 \%)$ & $0(0 \%)$ & $1(0.8 \%)$ \\
\hline Thrombocytopenia & $0(0 \%)$ & $0(0 \%)$ & $0(0 \%)$ & $1(0.8 \%)$ \\
\hline Tremor & $1(0.8 \%)$ & $0(0 \%)$ & $0(0 \%)$ & $1(0.8 \%)$ \\
\hline Vertigo & $1(0.8 \%)$ & $0(0 \%)$ & $2(1.5 \%)$ & $1(0.8 \%)$ \\
\hline Excessive Salivation & $1(0.8 \%)$ & $5(3.8 \%)$ & $41(31.3 \%)$ \\
\hline Total & $34(26.0 \%)$ & & \\
\hline
\end{tabular}

Note: Subjects with multiple adverse events in the same category are counted only once in that category; subjects with adverse events in $>1$ category are counted once for each type of severity.

\section{Effect of Blood Pressure and Arrhythmia after Pilocarpine Therapy}

Systolic and diastolic blood pressures and the pulse rate were evaluated for safety purposes at baseline and at 12 and 24 weeks after pilocarpine therapy, and overall, patients' vital signs assessed were not significantly changed by the study medication. Mean changes in systolic blood pressure from baseline were -0.4 and $-0.7 \mathrm{mmHg}$ after 12 and 24 weeks of treatment, respectively. Mean changes in diastolic blood pressure from baseline were - 0.2 and $-0.2 \mathrm{mmHg}$ after 12 and 24 weeks of treatment, respectively. However, those changes were not clinically relevant. Mean values of the pulse rate at 12 and 24 weeks were similar to those at baseline. No intervention was performed in the patients with palpitations, as no clinically significant abnormality was detected by 12-lead electrocardiography.

\section{Discussion}

The clinical effects of pilocarpine have been proven in many studies. Our study demonstrated the safety of pilocarpine for treating patients with Sjögren's syndrome. No SAEs were observed in this study. Although the single-arm post-marketing surveillance design limited the strength of the evidence, the data of 131 patients indicated the treatment benefits of pilocarpine in patients with Sjögren's syndrome. Sweating is the most common AE associated with pilocarpine treatment, and it could be the main reason for withdrawal from the study [17,19-21]. In a fixed-dose trial, sweating was reported in $43 \%$ of the patients during the 12 -week treatment period [20]. In a dose-titration study, pilocarpine was administered at a dose of $5 \mathrm{mg}$ QID for 6 weeks and then increased to $7.5 \mathrm{mg}$ QID for 6 weeks; sweating occurred in $64 \%$ of patients receiving pilocarpine treatment compared to $7 \%$ in the placebo group [22]. In a study conducted in the National Taiwan University Hospital, 5 patients $(21.7 \%)$ in the pilocarpine group experienced sweating, and 3 of them withdrew from the study [19]. A stepup dosing study in Japan found that $39.3 \%$ of subjects receiving pilocarpine experienced sweating [23]. Adequate patient education for sweating may be important for improving patient compliance after pilocarpine treatment.

The second common AE reported in our study was palpitation. Despite the higher rate of palpitation recorded in our study, the physical examinations and 12-lead electrocardiography did not detect any clinically relevant abnormality in patients with palpitation. The AEs of pilocarpine decreased over time. The frequency of AEs due to pilocarpine treatment may be reduced by starting with a low dose (e.g., $5 \mathrm{mg}$ once or twice daily), and then increasing the dose gradually until the maintenance dose of $5 \mathrm{mg}$ QID is achieved. According to a previous study, gastrointestinal adverse events caused by pilocarpine treatment tended to decrease over time, but not for sweating [23]. The dosing frequency used in our study ranged from once daily to QID. Among the 14 patients who experienced sweating in our study, 8 continued study treatment. The symptom lasted for more than 1 month in all 8 patients. The $\mathrm{AE}$ of sweating might have been tolerable for at least half of our patients. Pilocarpine acts like muscarinic cholinergic receptors found throughout the body and promotes fluid secretion. The salivary glands are stimulated, and the production of all the body's exocrine glands is increased. In contrast to pilocarpine, another drug for xerostomia called cevimeline has a high affinity for specific muscarinic receptors (M3) located in lachrymal and salivary gland epithelia. Ramos-Casals et al. reviewed controlled trials on drug therapy for primary Sjögren's syndrome and found four placebocontrolled trials that evaluated oral cevimeline in 376 patients [15].

The largest trial showed that $18 \%$ and $21 \%$ of subjects receiving cevimeline (30 mg three times daily) experienced sweating and nausea, respectively, and the second largest trial showed respective $\mathrm{AE}$ rates of $16 \%$ and $20 \%$ [15]. Although the treatment periods of these two studies (12 and 6 weeks) were much shorter than ours, the frequency of sweating in each trial was higher than that observed in our study. The third trial showed that $28 \%$ of subjects receiving cevimeline (30 $\mathrm{mg}$ three times daily) experienced diarrhea, and the last one reported that $14 \%$ of subjects had 
sweating, $19 \%$ had gastrointestinal side effects, and $24 \%$ had palpitation. These findings showed that gastrointestinal AEs were more prevalent in patients receiving cevimeline treatment than in those receiving pilocarpine treatments. Although the incidence of sweating was low in our study, some authors found that sweating was experienced more frequently with pilocarpine than with cevimeline [24]. Based on the assumption of the distinctive safety profiles between pilocarpine and cevimeline, the eligible subjects in the Japanese study were divided into four groups, with two groups receiving pilocarpine first and then cevimeline and vice versa. The frequencies of AEs including digestive symptoms and increased sweating decreased significantly in those two groups [23]. Thus, prescribing either pilocarpine or cevimeline is beneficial to patients with Sjögren's syndrome. However, patients must be warned of the potential AEs before receiving treatment with pilocarpine or cevimeline.

\section{Conclusion}

The most common AEs associated with pilocarpine treatment were sweating, palpitation, diarrhea, dizziness, nausea, epigastric discomfort, and headache. No serious AEs occurred during the study period. The safety profile observed herein may serve to remind clinical physicians of the essentials of appropriate patient education and monitoring. Pilocarpine therapy is safe, and it may be well tolerated by patients with Sjögren's syndrome.

\section{Acknowledgment}

This study was supported by the Taichung Armed-Forces General Hospital (grant number 107A7).

\section{References}

1. Jankowska AK, Waszkiel D, Kowalczyk A (2007) Saliva as a main component of oral cavity ecosystem. Part I. Secretion and function. Wiad Lek 60: 148-154.

2. Navazesh M, Kumar SK (2009) Xerostomia: prevalence, diagnosis, and management. Compend Contin Educ Dent 30: 326-328.

3. Mandel ID, Wotman S (1976) The salivary secretions in health and disease. Oral Sci Rev pp. 25-47.

4. Ferguson MM (1993) Pilocarpine and other cholinergic drugs in the management of salivary gland dysfunction. Oral Surg Oral Med Oral Pathol 75: 186-191.

5. Vanderas AP (1986) Bacteriologic and nonbacteriologic criteria for identifying individuals at high risk of developing dental caries: a review. J Public Health Dent 46: 106-113.

6. Borchers AT, Naguwa SM, Keen CL, Gershwin ME (2003) Immunopathogenesis of Sjogren's syndrome. Clin Rev Allergy Immunol 25: 89-104.

7. Kruszka P, O’Brian RJ (2009) Diagnosis and management of Sjögren syndrome. Am Fam Physician 79: 465-470.
8. Pillemer SR, Matteson EL, Jacobsson LT (2001) Incidence of physiciandiagnosed primary Sjögren syndrome in residents of Olmsted County, Minnesota. Mayo Clin Proc 76: 593-599.

9. Garcia-Carrasco M, Ramos-Casals M, Rosas J (2002) Primary Sjögren syndrome: clinical and immunologic disease patterns in a cohort of 400 patients. Medicine (Baltimore) 81: 270-280.

10. Stefanski AL, Tomiak C, Pleyer U (2017) The diagnosis and treatment of Sjögren's syndrome. Dtsch Arztebl Int 114: 354-361.

11. Serrano J, Lopez-Pintor RM, Gonzalez-Serrano J (2018) Oral lesions in Sjogren's syndrome: a systematic review. Med Oral Patol Oral Cir Bucal 23: e391-e400.

12. Tsigalou C, Stavropoulou E, Bezirtzoglou E (2018) Current insights in microbiome shifts in Sjogren's syndrome and possible therapeutic interventions. Front Immunol 9: 1106.

13. Mariette X, Criswell LA (2018) Primary Sjögren's syndrome. N Engl J Med 378: 931-939.

14. Wang SQ, Zhang LW, Wei P, Hua H (2017) Is hydroxychloroquine effective in treating primary Sjogren's syndrome: a systematic review and metaanalysis. BMC Musculoskelet Disord 18: 186.

15. Ramos-Casals M, Tzioufas AG, Stone (2010) Treatment of primary Sjögren syndrome: a systematic review. JAMA 304: 452-460.

16. Abreu IN, Andreazza NL, Sawaya AC (2007) Cell suspension as a tool to study the biosynthesis of pilocarpine in Jaborandi. Plant Biol (Stuttg) 9: 793-799.

17. Johnson JT, Ferretti GA, Nethery WJ (1993) Oral pilocarpine for postirradiation xerostomia in patients with head and neck cancer. $\mathrm{N}$ Engl J Med 329: 390-395.

18. Vivino FB (2001) The treatment of Sjögren's syndrome patients with pilocarpine-tablets. Scand J Rheumatol Suppl 115: 1-9.

19. Wu CH, Hsieh SC, Lee KL (2006) Pilocarpine hydrochloride for the treatment of xerostomia in patients with Sjögren's syndrome in Taiwan-a double-blind, placebo-controlled trial. J Formos Med Assoc 105: 796803.

20. Vivino FB, Al-Hashimi I, Khan Z (1999) Pilocarpine tablets for the treatment of dry mouth and dry eye symptoms in patients with Sjögren syndrome: a randomized, placebo-controlled, fixed-dose, multicenter trial. P92-01 Study Group. Arch Intern Med 159: 174-181.

21. Noaiseh G, Baker JF, Vivino FB (2014) Comparison of the discontinuation rates and side-effect profiles of pilocarpine and cevimeline for xerostomia in primary Sjögren's syndrome. Clin Exp Rheumatol 32: 575577.

22. Papas AS, Sherrer YS, Charney M (2004) Successful treatment of dry mouth and dry eye symptoms in Sjögren's syndrome patients with oral pilocarpine: a randomized, placebo-controlled, dose-adjustment study. J Clin Rheumatol 10: 169-177.

23. Shinozaki S, Masafumi M, Hayashida JN, Tanaka A (2015) A study of the effectiveness and side effects of salivation-inducers in patients with Sjögren's syndrome. Comparison between cevimeline hydrochloride and pilocarpine hydrochloride. Japanese Journal of Oral and Maxillofacial Surgery 61: 147-153.

24. Brimhall J, Jhaveri MA, Yepes JF (2013) Efficacy of cevimeline vs. pilocarpine in the secretion of saliva: a pilot study. Spec Care Dentist 33: 123-127. 
ISSN: 2574-1241

DOI: 10.26717/BJSTR.2019.13.002444

Hsiang-Cheng Chen. Biomed J Sci \& Tech Res

(C) (i) This work is licensed under Creative

Submission Link: https://biomedres.us/submit-manuscript.php

$\begin{array}{ll}\text { BIOMEDICAL } & \text { Assets of Publishing with us } \\ \text { RESEARCHES } & \text { Global archiving of articles } \\ \text { - Immediate, unrestricted online access }\end{array}$

